科 学 通 报

\title{
$\mathrm{Ge}(\mathrm{Li})$ 探测器 $\gamma$ 射线探测效率的新精确式
}

\author{
冯锡 璋 \\ (中国科学院高能物理研究所核应用部, 北京 100080)
}

\section{关键词 $\boldsymbol{\gamma}$ 射线探测效率}

在 $\gamma$ 谱学的研究和应用中, 常需知道 $\gamma$ 探测器对不同能量 $\left(E_{\gamma}\right)$ 的 $\gamma$ 射线的探 测效率 $\left(\varepsilon_{\gamma}^{0}\right)$ 或相对探测效率 ( $\varepsilon_{\gamma}$, 例如以 ${ }^{152} \mathrm{Eu}$ 的 $1408.08 \mathrm{keV} \gamma$ 的 $\varepsilon_{\gamma}=1.00 \%$ 为相对标准). 通常以 $\log E_{\gamma}$ 的函数, 如多项式, 表示 $\log \varepsilon_{\gamma}^{0[1]}$, 但这种方式因不能表达测量的全部物理过 程而不够精确. 因此, 我们已提出了两个能较好地表达测量的全部物理过程的新式 ${ }^{[2,3]}$. 但是, 为了进一步提高精确度, 我们再提出了一个新精确式以更好地表达 $\varepsilon_{r}^{0}$ 与 $E_{r}$ 之间的关系.

\section{1 理 论}

在一定的样品与探测器的几何排列下, 由样品发射出来的 $\gamma$ 射线部分通过样品及塑料容 器、空气层、薄窗和 $\mathrm{Ge}(\mathrm{Li})$ 探测器的不灵敏层等进入灵敏区, 产生光电过程或多次过程, 沉积 其全部能量而形成全吸收峰, 在 $63-1700 \mathrm{keV}$, 甚至更大一些的 $E_{r}$ 范围内, $\varepsilon_{r}^{0}$ 可用下式表 示:

$$
\varepsilon_{r}^{0}=G \cdot T_{i}(x) \cdot A_{s}(x) \cdot F(x),
$$

式中 $G$ 为几何因子; $T_{i}(x)$ 为光子通过样品及塑料容器、空气层、薄窗和 $\mathrm{Ge}(\mathrm{Li})$ 探测器的不 灵敏层等的穿透几率, $A_{\mathrm{s}}(x)$ 为光子在灵敏区中的吸收几率和 $F(x)$ 为在灵敏区被吸收光 子通过光电效应和多次过程 (如 Compton 效应与次级光子的光电效应)而产生全吸收过程的 几率,它们都是 $x$ 的函数, 而 $x=E_{r}^{-1[4]}$.

$$
T_{i}(x)-\exp \left[-\sum_{i} \mu_{i j}(x) d_{i i}\right],
$$

式中 $\mu_{i j}(x)$ 为光子在 $j$ 不灵敏吸收层中的吸收系数, $d_{i j}$ 为该层的平均厚度.

$$
A_{\mathrm{s}}(x)=1-\exp \left[-\mu_{\mathrm{s}}(x) d_{\mathrm{s}}\right] \text {, }
$$

式中 $\mu_{\mathrm{s}}(x)$ 为光子在灵敏区中的吸收系数, $d_{\mathrm{s}}$ 为该区的平均厚度.

$$
F(x)=\left[\mu_{\mathrm{s}, \mathrm{p}}(x)+a(x) \int_{\mu(2+\mu x)^{-1}}^{x^{-1}} \mu_{\mathrm{s}, \mathrm{c}}(x, k) \mu_{\mathrm{s}, \mathrm{p}}(k) d k\right] / \mu_{\mathrm{s}}(x),
$$

式中 $\mu$ 为电子静止质量, $\mu_{\mathrm{s}, \mathrm{p}}(x)$ 和 $\mu_{\mathrm{s}, \mathrm{p}}(k)$ 为在灵敏区中的光电效应吸收系数, $\mu_{\mathrm{s}, \mathrm{c}}(x, k)$ $d k$ 为在灵敏区中由 Compton 效应产生能量位于 $k$ 与 $k+d k$ 之间的次级光子的吸收系数, $a(x)$ 为比例因子. 对一定几何排列下的样品和一般 $\mathrm{Ge}(\mathrm{Li})$ 探测器, $G \cdot d_{i j}$ 和 $d_{s}$ 均为常 数, 各吸收系数及式 (4) 中的定积分, 并因此 $T_{i}(x) 、 A_{\mathrm{s}}(x)$ 与 $F(x)$ 都可用 $x$ 的多项式表 示 ${ }^{[4]}$. 所以, 在 $x-x_{m}$ 处具有一个极大值, 并且比较对称的 $\varepsilon_{r}^{0}=\varepsilon_{r}^{0}(x)$ 可用 $x$ 的多项式较 
好地表示 ${ }^{[2]}$. 但该式对能量较低的 $\boldsymbol{r}$ 射线 $\left(E_{r}<100 \mathrm{keV}\right)$ 的 $8_{r}^{0}$ 的表达并不完美. 因此,我 们考虑对该式作适当的改进.

根据实验和理论的知识,式 (2) 中的 $T_{i}(x)$ 可以较好地用 $\exp [f(x)]$ 表示,当 $\boldsymbol{E}_{\boldsymbol{r}}$ 较小 而 $x$ 较大时, 它是一个随着 $x$ 的增加而迅速下降但仍大于零的函数, 而 $f(x)$ 则是一个 $x$ 的多 项式. 这 $A_{a}(x) \cdot F(x)-g(x)$ 则可用一个 $x$ 的多项式表示, 它是一个随着 $x$ 的增加缓慢上 升的函数. 所以,

$$
\varepsilon_{r}^{0}-g \varepsilon_{r}-q \cdot g(x) \cdot \exp [f(x)] .
$$

这样, $\varepsilon_{r}^{0}$ 是一个 $x$ 的连续函数, 在 $x \geqslant 0$ 时, $\varepsilon_{r}^{0}>0$ 并只具有一个极大值.

\section{2 应用}

根据实验结果, 我们首先采用 $f(x)-b x^{\rho}$, 这里, $b<0$ 和 $\rho$ 为参数. 因此最简单的表 达式为

$$
\varepsilon_{\gamma}^{0}-q \varepsilon_{\gamma}-q \cdot\left(a_{0}+a_{1} x\right) \exp \left[b x^{\rho}\right],
$$

从 $x=15.4 \mathrm{MeV}^{-1}$ 实验结果 ${ }^{[}$, 得出 $b--0.0054, \rho-2.2, a_{0}-0$ 和 $a_{1}-1.4$. 采用 这些参数, 式 (5) 能较好地表达文献 [ 5 ]中的实验结果. 然后, 以最小二乘法将表 1 中文献 [5]的 ${ }^{152} \mathrm{Eu},{ }^{140} \mathrm{La},{ }^{160} \mathrm{~Tb},{ }^{182} \mathrm{Ta},{ }^{169} \mathrm{Yb}$ 和 ${ }^{131} \mathrm{Ba}$ 的 $E_{r}$ (以 $\mathrm{MeV}$ 表示)与 $\varepsilon_{r}(\%)$ 数据用下式

$$
y-\varepsilon_{\gamma} x^{-1} \exp \left(0.00540 x^{2.20}\right)-a_{0} x^{-1}+a_{1}+a_{2} x
$$

进行拟合而得出 $a_{0}, a_{1}, a_{2}$ 及式 (7) ${ }^{[3]}$

$$
\varepsilon_{r}-\left(0.0 .94387+1.305272 x+0.014133 x^{2}\right) \exp \left(-0.00540 x^{2.20}\right) \text {. }
$$

更进一步提出 $f(x)-b_{3} x^{3}+b_{2} x^{2}+b_{1} x+b_{0}$ 和 $\varepsilon_{\gamma}-\left(0.094387+1.305272 x+0.014133 x^{2}\right)$ - $\exp \left(-b_{0}\right) \exp [f(x)]$, 再以最小二乘法将上面六个核素的 $E_{\boldsymbol{r}}$ (以 $\mathrm{MeV}$ 表示)与由式(7)得 出的 $\varepsilon_{r}(\%)$ 值用下式

$$
y-x^{-2} \ln \left[\varepsilon_{r}\left(0.094387+1.305272 x+0.014133 x^{2}\right)^{-1}\right]-b_{3} x+b_{2}+b_{1} x^{-1}
$$

进行拟合而得出 $b_{1}, b_{2}, b_{3}, f(x)$ 和新精确式

$$
\begin{gathered}
f(x)=-0.000169 x^{3}-0.00685 x^{2}+0.00114 x+b_{0}, \\
\varepsilon_{\gamma}=\left(0.094387+1.305272 x+0.014133 x^{2}\right) \exp \left(-0.000169 x^{3}-0.00685 x^{2}+0.00114 x\right),
\end{gathered}
$$

这里, $b_{0}$ 值的大小决定于各不灵敏层的性质. 由于式(8)中不包含 $b_{0}$, 所以 $b_{0}$ 值的大小对 $\varepsilon_{\gamma}$ 值无影响而只影响 $f(x)$ 和 $T_{i}(x)$ 值. 又由于 $\exp \left(b_{0}\right)$ 为一常数, 所以它不影响 $T_{i}(x)$ 的形状. 若取 $b_{0}=-0.00200$, 我们可以看到在 $x-0.083 \mathrm{MeV}^{-1}\left(E_{\gamma}=12.0 \mathrm{MeV}\right)$ 处, $T_{i}(x)-\exp [f(x)]$ 具有一个极大值 0.998. 这是由于光电效应、Compton 效应与电子对产 生三种光子吸收机理的综合结果. 根据文献[6], 对 $\mathrm{C}, \mathrm{Al}, \mathrm{Cu}$ 和 $\mathrm{Sn}$, 这极大值应各在 $E_{r}$ 约为 $60,15,7$ 和 $5 \mathrm{MeV}$ 处出现, 那里,它们的光子吸收系数各具有极小值. 根据我们的实验 中各个不灵敏层的材料和厚度到, $T_{i}(x)$ 在 $E_{\gamma}=12 \mathrm{MeV}$ 处出现一个极大值与由实验条件 所作估计是相一致的. 在式 (8) 中, $\varepsilon_{r}$ 是一个 $x$ 的连续函数, 并在 $x \geqslant 0$ 时只具有一个极大 值.

在表 1 中,我们列出了文献 $[5,2]$ 和本文式 $(7)^{[3]}$ 与式(8)的结果. 这里, $f_{i j}$ 为在核素 $i$ 的一次衰变中发射第 $j$ 种 $\gamma$ 射线(简称 $j$ 光子, 后同)的几率; $B_{i j}^{0}-B_{i j} / q$ 为由 $j$ 光子的光峰 计数计算得出的每单位重量的某元索 $z_{i}$ 在中子照射过程中产生核素 $i$ 的速率. 从表 1 中, 
表 1 放射性核素的 $r$ 射线相对探测效率和相对生成速率

\begin{tabular}{|c|c|c|c|c|c|c|c|c|c|c|}
\hline \multirow{2}{*}{ (元素 } & \multirow{2}{*}{$\begin{array}{l}\boldsymbol{\gamma} \text { 能量 } \\
(\mathrm{keV})\end{array}$} & \multirow{2}{*}{$\begin{array}{c}r \text { 发射率 } \\
f_{i j}\end{array}$} & \multicolumn{4}{|c|}{$\begin{array}{c}\text { 相对探测效率(\%) } \\
\varepsilon_{r}\end{array}$} & \multicolumn{4}{|c|}{ 相对生成速率 $\left(B_{i} ;(\mathrm{s})^{-1} \cdot(\mathrm{g})^{-1}\right)$} \\
\hline & & & the & 文献 [ 2 & $\begin{array}{l}\text { 文献 [3] } \\
\text { 本文式(7) }\end{array}$ & $\mid$\begin{tabular}{|c|} 
本工作 \\
$\mid$ 文式 (8)
\end{tabular} & 文献 [ 5 ] & 文献 [2 ] & 文南 & 本文式 \\
\hline \multirow{5}{*}{$\begin{array}{c}\mathrm{Yh} \\
\left({ }^{109} \mathrm{Yb}\right)\end{array}$} & 63.12 & $4.38 \times 10^{-1}$ & 2.40 & 2.31 & 31 & 2.27 & $2.626 \times 10^{11}$ & $2.73 \times 10^{11}$ & $2.73 \times 10^{11}$ & $2.78 \times 10^{12}$ \\
\hline & 130.52 & $1.14 \times 10^{-1}$ & 6.80 & 6.90 & 6.78 & 6.83 & $3.328 \times 10^{11}$ & $3.28 \times 10^{11}$ & $3.34 \times 10^{11}$ & $3.31 \times 10^{11}$ \\
\hline & 177.21 & $2.17 \times 10^{-1}$ & 6.25 & 6.34 & 6.20 & 6.21 & $3.031 \times 10^{11}$ & $2.99 \times 10^{11}$ & $3.06 \times 10^{14}$ & $3.05 \times 10^{11}$ \\
\hline & 197.95 & $3.56 \times 10^{-1}$ & 5.95 & 5.93 & 5.83 & 5.82 & $2.839 \times 10^{11}$ & $2.85 \times 10^{11}$ & $2.90 \times 10^{12}$ & $2.90 \times 10^{11}$ \\
\hline & 307.73 & $9.97 \times 10^{-2}$ & 4.20 & 4.15 & 4.17 & 4.16 & $3.369 \times 10^{11}$ & $3.41 \times 10^{11}$ & $3.39 \times 10^{11}$ & $3.40 \times 10^{11}$ \\
\hline \multirow{4}{*}{$\begin{array}{c}\mathrm{La} \\
\left({ }^{140} \mathrm{La}_{\mathrm{a}}\right)\end{array}$} & 328.77 & $1.85 \times 10^{-1}$ & 3.90 & 3.91 & .94 & 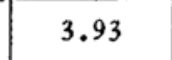 & $6.78 \times 10^{11}$ & $6.76 \times 10^{11}$ & $6.71 \times 10^{11}$ & $6.73 \times 10^{21}$ \\
\hline & 487.03 & $4.30 \times 10^{-1}$ & 2.77 & 2.72 & 2.76 & 2.76 & $6.40 \times 10^{11}$ & $6.52 \times 10^{11}$ & $6.42 \times 10^{11}$ & $6.42 \times 10^{1:}$ \\
\hline & 815.85 & $2.36 \times 10^{-1}$ & 1.63 & 1.69 & 1.701 & 1.700 & $7.24 \times 10^{11}$ & $6.98 \times 10^{11}$ & $6.94 \times 10^{12}$ & $6.94 \times 10^{11}$ \\
\hline & 1596.49 & $9.55 \times 10^{-1}$ & 0.88 & 0.93 & 0.916 & 0.916 & $6.83 \times 10^{11}$ & $6.46 \times 10^{11}$ & $6.56 \times 10^{11}$ & $6.56 \times 10^{11}$ \\
\hline \multirow{4}{*}{$\begin{array}{c}\mathrm{Ba} \\
\left({ }^{131} \mathrm{Ba}\right)\end{array}$} & 123.73 & $2.8 \times 10^{-1}$ & 6.80 & 6.85 & 6.77 & סנ.0 & $8.18 \times 10^{8}$ & $8.12 \times 10^{8}$ & $8.22 \times 10^{8}$ & $8.14 \times 10^{8}$ \\
\hline & 216.01 & $2.17 \times 10^{\cdots 1}$ & 5.65 & 5.58 & 5.50 & 5.50 & $7.91 \times 10^{8}$ & $8.01 \times 10^{8}$ & $8.13 \times 10^{8}$ & $8.13 \times 10^{\varepsilon}$ \\
\hline & 373.15 & $1.3 \times 10^{-1}$ & 3.30 & 3.48 & 3.52 & 3.52 & $1.013 \times 10^{9}$ & $9.61 \times 10^{8}$ & $9.50 \times 10^{8}$ & $9.50 \times 10^{8}$ \\
\hline & 496.23 & $4.21 \times 10^{-1}$ & 2.70 & 2.67 & 2.71 & 2.71 & $8.80 \times 10^{8}$ & $8.90 \times 10^{8}$ & $8.77 \times 10^{8}$ & $8.77 \times 10^{8}$ \\
\hline \multirow{7}{*}{$\begin{array}{c}\mathrm{Ta} \\
\left({ }^{182} \mathrm{~T} a\right)\end{array}$} & 67.75 & $4.14 \times 10^{-1}$ & 2.90 & 2.88 & 99 & >0 & $1.762 \times 10^{12}$ & $1.77 \times 10^{12}$ & $1.71 \times 10^{12}$ & $1.71 \times 10^{12}$ \\
\hline & 100.11 & $1.41 \times 10^{-1}$ & 6.40 & 5.98 & 6.19 & 6.27 & $1.478 \times 10^{12}$ & $1.58 \times 10^{12}$ & $1.53 \times 10^{12}$ & $1.51 \times 10^{12}$ \\
\hline & 152.43 & $7.2 \times 10^{-2}$ & 6.55 & 6.77 & 6.60 & 6.63 & $1.547 \times 10^{12}$ & $1.50 \times 10^{12}$ & $1.54 \times 10^{12}$ & $1.53 \times 10^{12}$ \\
\hline & 222.10 & $7.98 \times 10^{-2}$ & 5.50 & 5.46 & 5.40 & 5.39 & $1.451 \times 10^{12}$ & $1.46 \times 10^{12}$ & $1.48 \times 10^{12}$ & $1.48 \times 10^{12}$ \\
\hline & 1121.28 & $3.51 \times 10^{-1}$ & 1.30 & 1.27 & 1.264 & 1.264 & $1.484 \times 10^{12}$ & $1.52 \times 10^{12}$ & $1.53 \times 10^{12}$ & $1.53 \times 10^{12}$ \\
\hline & 1221.42 & $2.89 \times 10^{-1}$ & 1.20 & 1.17 & 1.168 & 1.168 & $1.463 \times 10^{12}$ & $1.50 \times 10^{12}$ & $1.50 \times 10^{12}$ & $1.50 \times 10^{12}$ \\
\hline & 1230.97 & $1.16 \times 10^{-1}$ & 1.20 & 1.17 & 1.160 & 1.160 & $1.448 \times 10^{12}$ & $1.49 \times 10^{12}$ & $1.50 \times 10^{12}$ & $1.50 \times 10^{12}$ \\
\hline \multirow{7}{*}{$\underset{\left({ }^{152} \mathrm{Eu} u\right)}{\mathrm{Eu}}$} & 244.67 & $7.0 \times 10^{-2}$ & 10 & 5.0 & & & & $1.68 \times 10^{14}$ & $1.69 \times 10^{14}$ & $1.69 \times 10^{14}$ \\
\hline & 344.30 & $2.72 \times 10^{-1}$ & 60 & 3.75 & 79 & 78 & $1.864 \times 10^{14}$ & $1.79 \times 10^{14}$ & $1.77 \times 10^{14}$ & $1.78 \times 10^{14}$ \\
\hline & 778.90 & $1.27 \times 10^{-1}$ & 70 & 1.76 & 777 & 775 & $1.755 \times 10^{14}$ & $1.70 \times 10^{14}$ & $1.68 \times 10^{14}$ & $1.68 \times 10^{14}$ \\
\hline & 963.97 & $1.43 \times 10^{-1}$ & 1.46 & 1.45 & 55 & 154 & $1.909 \times 10^{14}$ & $1.92 \times 10^{14}$ & $1.92 \times 10^{14}$ & $1.92 \times 10^{14}$ \\
\hline & 1085.80 & $1.01 \times 10^{-1}$ & 1.34 & 1.30 & 1.303 & 1.302 & $2.007 \times 10^{14}$ & $2.07 \times 10^{14}$ & $2.05 \times 10^{14}$ & $2.07 \times 10^{14}$ \\
\hline & 1112.07 & $1.34 \times 10^{-1}$ & 1.32 & 1.28 & 1.274 & 1.274 & $1.831 \times 10^{14}$ & $1.89 \times 10^{14}$ & $1.90 \times 10^{14}$ & $1.90 \times 10^{14}$ \\
\hline & 1408.08 & $2.07 \times 10^{-1}$ & 1.00 & 1.04 & 1.026 & 1.026 & $1.837 \times 10^{14}$ & $1.77 \times 10^{14}$ & $1.79 \times 10^{14}$ & $1.79 \times 10^{14}$ \\
\hline \multirow{5}{*}{$\begin{array}{c}\mathrm{Tb} \\
\left({ }^{160} \mathrm{~Tb}\right)\end{array}$} & 86.79 & $1.29 \times 10^{-1}$ & 4.90 & 4.86 & 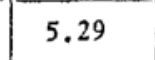 & 5.36 & $1.704 \times 10^{12}$ & $1.72 \times 10^{12}$ & $1.58 \times 10^{12}$ & $1.56 \times 10^{12}$ \\
\hline & 298.57 & $2.73 \times 10^{-1}$ & 4.35 & 4.26 & 4.28 & 4.27 & $1.512 \times 10^{12}$ & $1.54 \times 10^{12}$ & $1.54 \times 10^{12}$ & $1.54 \times 10^{12}$ \\
\hline & 879.37 & $3.0 \times 10^{-1}$ & 1.56 & 1.57 & 1.586 & 1.585 & $1.708 \times 10^{12}$ & $1.70 \times 10^{12}$ & $1.68 \times 10^{12}$ & $1.68 \times 10^{12}$ \\
\hline & 966.17 & $2.49 \times 10^{-1}$ & 1.46 & 1.45 & 1.452 & 1.451 & $1.570 \times 10^{12}$ & $1.58 \times 10^{12}$ & $1.58 \times 10^{12}$ & $1.58 \times 10^{1:}$ \\
\hline & 1177.60 & $2.06 \times 10^{-1}$ & 1.26 & 1.21 & 1.208 & 1.208 & $1.367 \times 10^{12}$ & $1.42 \times 10^{12}$ & $1.43 \times 10^{12}$ & $1.43 \times 10^{6}$ \\
\hline \multirow{2}{*}{$\underset{\left({ }^{147} \mathrm{Nd}\right)}{\mathrm{Nd}}$} & .11 & $2.80 \times 10^{-1}$ & 5.40 & 5.27 & & 72 & $2.08 \times 10^{10}$ & $2.13 \times 10^{10}$ & $1.99 \times 10^{20}$ & $1.96 \times 10^{10}$ \\
\hline & 531.60 & $1.28 \times 10^{-1}$ & 2.55 & 2.50 & 2.54 & 4 & 1.88 & $1.92 \times 10^{10}$ & $1.89 \times 10^{10}$ & 1.89 \\
\hline \multirow{2}{*}{$\begin{array}{c}\mathrm{Sc} \\
\left({ }^{46} \mathrm{Sc}\right)\end{array}$} & & & 1.54 & 1 & & & & $10^{12}$ & $10^{12}$ & 6.53 \\
\hline & 1120.52 & 1.00 & 1.30 & 1.27 & 265 & 65 & $5.58 \times 10^{12}$ & $6.38 \times 10^{12}$ & $6.40 \times 10^{12}$ & $6.40 \times 10^{12}$ \\
\hline \multirow{2}{*}{$\begin{array}{l}\mathrm{Co} \\
\left({ }^{60} \mathrm{Co}\right)\end{array}$} & 1 & & 0 & 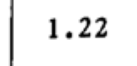 & 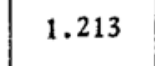 & 13 & $7.00 \times 10^{12}$ & $7.23 \times 10^{12}$ & $7.27 \times 10^{12}$ & 7.27 \\
\hline & 1332.46 & 1.00 & 1.08 & 1.09 & 079 & .079 & $7.17 \times 10^{12}$ & $7.10 \times 10^{12}$ & $7.18 \times 10^{12}$ & $7.18 \times 10^{12}$ \\
\hline \multirow{2}{*}{$\begin{array}{c}\mathrm{Ho} \\
\left({ }^{166} \mathrm{Ho}\right)\end{array}$} & & & & & & & $6.28 \times 10^{12}$ & $6.56 \times 10^{12}$ & $5.93 \times 10^{12}$ & $5.85 \times 1.12$ \\
\hline & 1380.0 & & 1.04 & 1.06 & 45 & .045 & $5.58 \times 10^{12}$ & $5.47 \times 10^{12}$ & $5.55 \times 10^{12}$ & $5.55 \times 10^{12}$ \\
\hline
\end{tabular}


我们可以看到, 根据文献 $[5,2]$ 的标准, 由本文式 (7) $)^{[3]}$ 和式 (8) 得出的 $B_{i j}$ 和 $\varepsilon_{r}$ 优于文献 $[5,2]$ 的结果, 尤其是当 $E_{\gamma}<100 \mathrm{keV}$ 时. 对能量低的 $\gamma$ 射线, 式(8)稍优于式 (7); 对中、高 能 $\boldsymbol{r}$ 射线则二式很相近.

\section{3 讨 论}

从以上的结果, 我们可以看到 $\exp [f(x)]$ 是 $T_{i}(x)$ 的一个由拟合得出的并在由实验条 件估计出的 $E_{\gamma}$ 处有一个级大值的很精确的表达式. 所以, 根据表 1 中的结果和 $T_{i}(x)=$ $\exp [f(x)]$ 的优越性, 式(5)和式(8)确是 $\varepsilon_{\boldsymbol{r}}$ 的新精确式, 并且很可能是目前一般 $\mathrm{Ge}(\mathrm{Li})$ 探 测器的 $\varepsilon$, 能最佳表达式.

致谢作者对 King College 物理系 E. W. Jr. Burke 教授的热情帮助表示感谢。

\section{参考文嗝}

[1] 刘承安、汤乃勋、冯锡璋,核技术, 1984,(6): 21-26.

[2]冯锡璄、孙建国,科学通报, 1989,34 (10): 737-740.

[3] Feng, X., Journal of Radioanalytical and Nuclear Chcmistry. 1991, 151 (1):41-46.

[4] Heitler, W., The Quantum Theory of Radiation, 3rd edition. 1954, V.

[5] 冯锡墇、罗世华、季国坤等, 北京师范大学学报(自然科学版), 1984，(3): 59-68.

[6] Friedlander, G. et al., Nuclear and Radiochemistry, 3rd edition, Chapters 6-7, 1981. 